\title{
Crise et gestion de crise Application à l'eau
}

\author{
par Michel Mesny \\ Ingénieur général du Génie rural, des Eaux et des Forêts
}

\section{INTRODUCTION}

Crise et gestion de crise constituent un sujet essentiel, car lié aux vulnérabilités de nos sociétés modernes. En effet, nos sociétés se caractérisent par une sophistication, une spécialisation, une urbanisation, une mondialisation et une médiatisation qui les rendent vulnérables.

Le citoyen y a besoin, pour vivre comme pour jouer son rôle d'agent économique producteur, d'être raccordé à des réseaux de service (énergie, eau potable et eaux usées, télécommunications, monétique, etc...). Or ces réseaux sont vulnérables par leur étendue et par leurs ramifications, bien que leur maillage puisse apporter un élément de sécurité. Il suffit d'un grain de sable dans un rouage, un virus informatique par exemple, pour que la machine s'arrête. C'est ainsi qu'en juillet 1992 le blocus des grands axes et des points stratégiques par les chauffeurs routiers a failli empêcher la production d'eau potable par manque d'eau de javel... On n'avait pas pensé à une telle conséquence.

\section{II — VULNÉRABILITÉ}

Cette complexité, cette sophistication, cette spécialisation, et cette mondialisation nécessitent pour les gérer une organisation stricte : l'organisation est le maître mot de nos sociétés développées. Dans le même temps, une organisation économiquement optimale s'accompagne souvent de flux tendus, qui augmentent les vulnérabilités.

L'entropie est la grandeur qui quantifie le désordre et elle augmente avec lui ; à un ordre parfait correspond une entropie nulle. L'entropie a tendance à augmenter spontanément et une crise correspond à un saut d'entropie. Pour réduire et maintenir à un niveau bas l'entropie d'un système, il est nécessaire de dépenser beaucoup d'énergie : un système organisé est donc un système instable et par conséquent vulnérable. Autant il est difficile d'organiser, autant il est facile de désorganiser... d'autant plus que des progrès importants ont été faits dans le savoir-faire de la désorganisation. Si le 7 décembre 1941 la destruction de la flotte américaine à Pearl Harbour par les Japonais a été une opération très lourde, le 16 janvier 1991 pendant la guerre du Golfe, il a suffit de quelques frappes chirurgicales pour détruire le système de commandement et les radars de l'armée de l'air irakienne, la rendant non opérationnelle.

\begin{abstract}
A l'inverse, nos sociétés développées sont désemparées pour lutter contre des adversaires désorganisés : le problème des banlieues, de la Palestine dans les territoires qui étaient occupés, avec des forces de maintien de l'ordre désemparées et inefficaces devant les jets de pierre, en sont des exemples. En effet, on ne peut pas désorganiser ce qui n'est pas organisé. C'était le problème en Bosnie où les casques bleus et les forces de l'OTAN étaient "désarmés" devant les milices et les snippers ; même chose en Somalie en 1993, où les soldats américains ont dû battre en retraite face aux bandes du seigneur de guerre, Mohamed Farrah Aidid. Cette vulnérabilité de nos sociétés modernes est encore aggravée par le fait que les situations ne sont plus "noir ou blanc" ( guerre ou paix - amis ou ennemis), mais que les situations et aussi les zones deviennent grises avec multiplication de risques diffus. Cette nouvelle donne nécessite une vigilance permanente, grande consommatrice d'énergie.
\end{abstract}

\section{III — DÉMARCHE SÉCURITAIRE}

Face à cette vulnérabilité et à ces risques de crise, une démarche sécuritaire globale s'impose.

Cette démarche sécuritaire comprend quatre volets :

1/ Connaître les menaces et les risques, et déterminer les vulnérabilités. En ce qui concerne les risques industriels, ce sont les études de dangers : il faut connaître et faire connaître.

2/ Une fois connues, les vulnérabilités doivent être réduites par des dispositifs de protection et de sécurisation et par l'élaboration de solutions de secours qu'il y a lieu de tenir en réserve. Dans le cas des risques industriels, ce sont les Plans d'Opération Interne (P.O.I.).

3/ Les mesures de prévention ne pouvant pas toujours empêcher le déclenchement de la crise, la gestion de crise doit être préparée par la mise au point de plans d'urgence, qu'il est nécessaire de s'entraîner à appliquer par des exercices. En matière de risques industriels, ce sont les Plans Particuliers d'Intervention (P.P.I.).

4/ Des détecteurs doivent être mis en place afin d'avertir de tout dérèglement risquant de conduire à une crise, ou d'alerter du déclenchement même de la crise de façon à la gérer dès qu'elle survient. 


\section{IV — SÉCURITÉ DE L'ALIMENTATION EN EAU POTABLE}

- 4.1 Réflexion sur la sécurité de l'alimentation en eau potable

Assurer la continuité et la fiabilité de la distribution d'eau potable aux populations, c'est un défi que relèvent au quotidien les distributeurs d'eau. Les réseaux modernes sont en général redondants, souvent interconnectés et permettent de limiter la portée des incidents, si bien que les défaillances passent généralement inaperçues pour le consommateur. Mais lorsque survient l'accident grave, c'est toute la population qui est directement menacée. Nous donnerons deux exemples :

— Lima au Pérou en 1991 : 10000 morts par choléra à cause de la suppression de la chloration de l'eau potable, - Milwaukee (USA) au printemps 1993 : 100 morts à cause d'un protozoaire parasite, le cryptosporidium, dans l'eau potable.

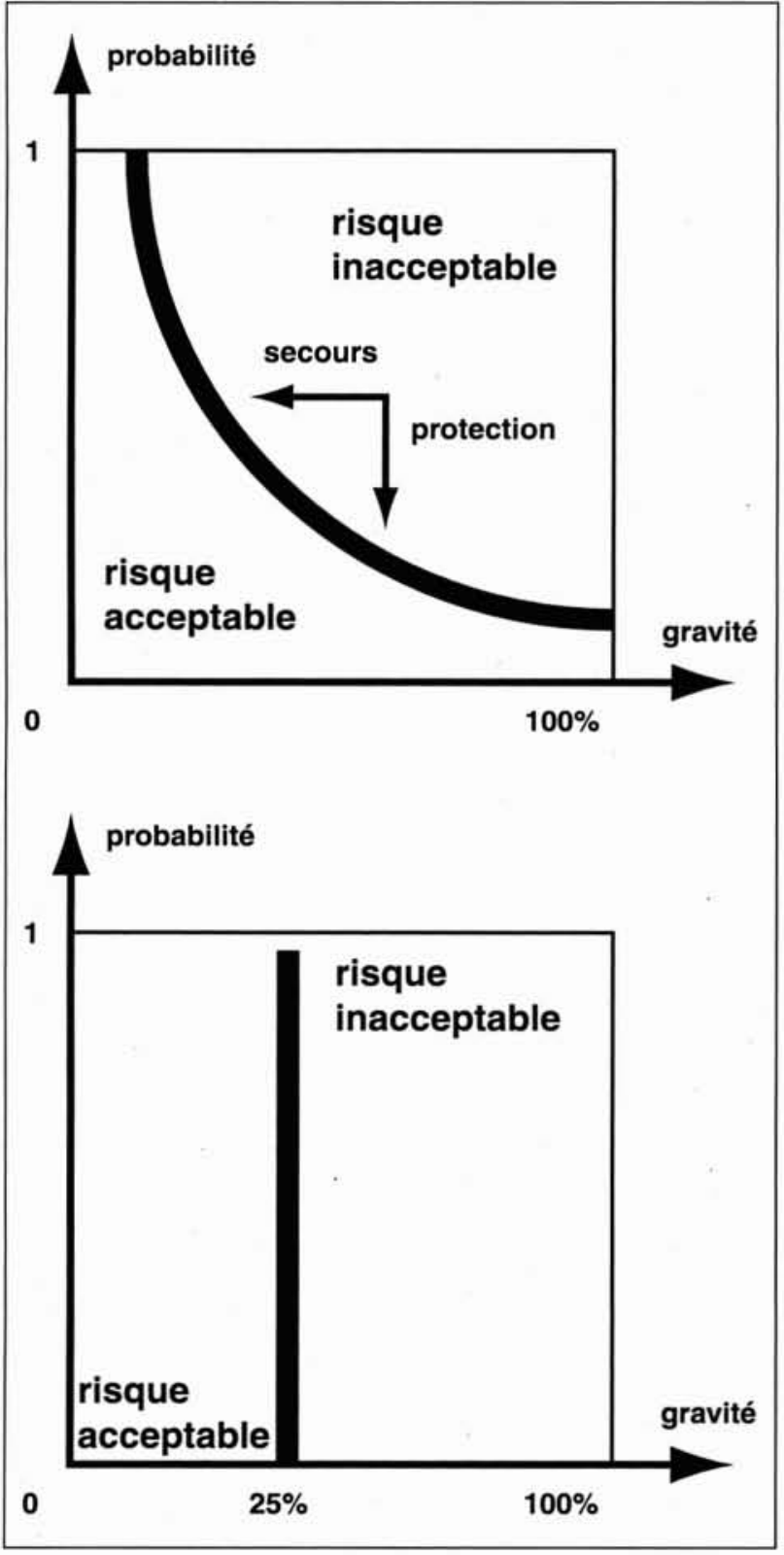

1. Courbes d'égale vulnérabilité en coordonnées "gravité" et "probabilité".

\subsubsection{Vulnérabilité}

La vulnérabilité (ou sensibilité) d'un réseau d'alimentation en eau potable par rapport à un de ses composants peut être quantifiée en fonction des caractéristiques de la défaillance de l'élément considéré. Cette vulnérabilité est le produit de deux facteurs :

Vulnérabilité $=$ gravité $\times$ probabilité

- Le facteur gravité mesure les conséquences de la mise hors-service de l'élément considéré et peut être facilement calculé en pourcentage de la réduction du service. Ce facteur gravité diminue si des solutions de secours opérationnelles existent.

- Le facteur probabilité de mise hors-service ou de dégradation est difficile à calculer, car il intègre les taux de défaillance, les délais de réparation et il dépend de perturbations souvent mal définies. Ce facteur probabilité diminue si des dispositifs de protection sont en place. Il est faible pour les conduites enterrées, plus important pour les réservoirs d'eau potable à surface libre.

En coordonnées "gravité" et "probabilité", les courbes d'égale vulnérabilité sont des hyperboles. Une de ces courbes définit une vulnérabilité acceptable, donc des plages de risque acceptable/inacceptable (Figure 1).

La vulnérabilité d'un réseau est celle de ses composants les plus vulnérables, pondérée par le nombre d'éléments appartenant à la plage de vulnérabilité supérieure. Cependant, compte tenu de la difficulté d'apprécier le facteur probabilité, il est proposé d'évaluer la vulnérabilité d'un réseau en se limitant au facteur gravité : on recherche les constituants du réseau, y compris les ressources en eau, dont la panne, la dégradation ou l'indisponibilité entraînerait le plus grand pourcentage de réduction du service par rapport à la demande journalière maximale. Comme on ne considère que la mise hors-service d'élément un par un et non simultanément, la capacité résiduelle du réseau doit rester élevée. La norme serait qu'aucun constituant du réseau ne commande plus de $25 \%$ de la demande, laissant, en cas d'indisponibilité ou de mise hors-service de ce seul élément, $75 \%$ de capacité résiduelle grâce aux autres éléments du réseau ou à l'activation des solutions de secours existantes. Cette approche illustrée par la deuxième courbe de la figure 1 doit être menée pour les ressources en eau et pour le réseau avec ses ouvrages.

\subsubsection{Points sensibles et service minimum}

Cette analyse de la vulnérabilité d'un réseau d'alimentation en eau potable par rapport à ses composants conduit à la notion de points sensibles ("key points" chez les britanniques). Il existe en France trois catégories de Points Sensibles, formalisées par le Secrétariat général de la Défense nationale : PS1 ou indispensable, PS2 ou nécessaire, PS3 ou utile.

En matière d'eau potable, la classification en point sensible est déterminée en fonction de la zone desservie (Paris, métropoles d'équilibre, villes de plus de 50000 habitants) et de l'importance relative de l'installation considérée. Cette importance est mesurée par la gravité de la mise hors-service de l'installation, c'est-à-dire par la réduction de la capacité du réseau, en pourcentage de la consommation journalière maximale d'eau potable (plus de $50 \%$, de 35 à $50 \%$, de 25 à $35 \%$ ), ou éventuellement en pourcentage de la population ne pouvant plus être desservie (Figure 2, page suivante) ; on tient compte de l'activation des solutions de secours existantes. Pour prendre l'exemple de Paris, la mise hors-service de l'élément le plus important du réseau, le double aqueduc de la Vanne et du Loing, n'entraînerait qu'une réduction de $17 \%$ du service maximal grâce à la diversification des sources d'alimentation de la capitale. 
Cependant, la défaillance des installations étant considérée une par une et leur gravité pouvant rester faible lorsqu'il y a redondance des capacités de production et de secours, les critères précédents peuvent ne pas entraîner de classement en point sensible. Aussi peut-il alors être proposé, spécialement pour les plus grandes villes, le classement d'une ou plusieurs installations stratégiques qui soient les mieux à même d'assurer un service minimum en cas de défaillances multiples. Alors que les besoins de survie sont d'environ 4 litres par jour et par habitant, le service minimum qu'il est souhaitable de maintenir ou de rétablir rapidement pour assurer un niveau d'activité économique acceptable est de 100 litres par jour et par habitant, à comparer avec un service normal d'environ 250 litres. A Paris, les conséquences multiples d'une crue de type 1910 de la Seine et de ses affluents laisseraient opérationnel $80 \%$ du service moyen (voir paragraphe 4.5 ).

\subsection{Risques et menaces pour l'alimentation en eau potable}

La détermination des vulnérabilités correspond au premier volet de la démarche sécuritaire globale détaillée en III. L'alimentation en eau potable est constituée :

- de ressources en eau,

- d'une ou plusieurs stations de traitement,

- de réseaux de distribution avec des ouvrages (réservoirs, stations de pompage) et des conduites.

\subsection{Ressources en eau}

Les ressources en eau peuvent être affectées par :

— des risques naturels :

- sécheresse avec des problèmes de quantité et de qualité, tels qu'eutrophisation et développement d'algues,

- inondations pouvant accroître la turbidité de l'eau et entraîner la submersion des installations situées en bord de rivière.

- des risques technologiques :

- une pollution accidentelle peut obliger à arrêter le traitement, donc la production d'eau potable.

Les sources et les eaux souterraines étaient traditionnellement les ressources privilégiées pour l'alimentation en eau potable, car de meilleure qualité et mieux protégées que les eaux superficielles. Cependant, la montée des teneurs en nitrates et en pesticides dans les eaux souterraines, l'amélioration des techniques de traitement et la sévérité accrue des normes, en particulier européennes, conduisent certaines collectivités à préférer les eaux de rivière, qui répondent plus vite aux efforts de dépollution, mais sont plus vulnérables parce qu'exposées aux pollutions accidentelles.

\subsubsection{Stations de traitement et réseaux}

Pour produire et distribuer de l'eau potable, il faut de l'électricité et des produits chimiques, et pour gérer les réseaux il faut des télécommunications. Ainsi en France, lors du blocus par les chauffeurs routiers en juillet 1992, certaines usines de traitement d'eau potable ont failli être en rupture de stock d'eau de javel et ainsi dans l'incapacité de produire de l'eau potable.

Des éléments du réseau peuvent être mis hors-service par des phénomènes naturels (inondations, ouragan, séisme) ou des actes de négligence, de malveillance, voire de terrorisme ou de guerre. Enfin, de façon plus sournoise, difficile à détecter, un réseau de distribution d'eau potable peut être contaminé, soit par des retours d'eau accidentels (en particulier dans le cas de branchements industriels), soit par des contaminations criminelles. Pour un germe présentant un pouvoir pathogène élevé et pour la toxine la plus virulente, les doses létales $50 \%$ (DL 50) pour l'homme peuvent des-
IMPORTANCE DE LA ZONE DESSERVIE

\begin{tabular}{|l|c|c|c|}
\hline & $\begin{array}{c}\text { PARIS } \\
\text { et région } \\
\text { parisienne }\end{array}$ & $\begin{array}{c}\text { Métropoles } \\
\text { d'équilibre }\end{array}$ & $\begin{array}{c}\text { Villes de plus } \\
\text { de 50 000 } \\
\text { habitants }\end{array}$ \\
\hline Coefficient a & 1 & 2 & 3 \\
\hline
\end{tabular}

IMPORTANCE RELATIVE DE L'INSTALLATION

(réduction de la capacité du réseau en pourcentage de la consommation journalière maximale d'eau potable).

\begin{tabular}{|c|c|c|c|}
\hline Gravité & plus de $50 \%$ & 35 à $50 \%$ & 25 à $35 \%$ \\
\hline Coefficient b & 1 & 2 & 3 \\
\hline
\end{tabular}

\section{CLASSEMENT EN POINTS SENSIBLES}

\begin{tabular}{|c|c|c|c|}
\hline & PS1 & PS2 & PS3 \\
\hline $\mathbf{a} \times \mathbf{b}$ & 1 & 2 & 3 et 4 \\
\hline
\end{tabular}

2. Organisation de la sécurité des réseaux d'eau potable : points sensibles.

cendre respectivement à $10^{3}$ germes et moins de 1 microgramme. Or du fait de leur étendue les réseaux d'eau potable sont difficiles à protéger, d'autant plus que les réservoirs, éléments particulièrement sensibles, sont souvent isolés.

Face à ces risques et menaces, la sécurité de l'alimentation en eau potable suppose :

- un état d'esprit permanent de vigilance de la part des distributeurs d'eau,

- la mise en place de dispositifs de sécurisation préventive des installations (protection et détection),

- l'existence de solutions de secours,

et si malgré tout la crise survient :

- la mise en cuvre de plans d'urgence.

\subsection{Mesures de protection et de sécurisation}

\subsubsection{Dispositifs de protection physique}

- Des fermetures de sécurité et des alarmes anti-intrusion doivent équiper tous les accès aux surfaces libres d'eau potable (réservoirs).

- Les branchements de distribution doivent être équipés de clapets anti-retour ou mieux de disconnecteurs (normalement obligatoires pour les branchements industriels) afin d'éviter tout retour d'eau contaminée dans le réseau d'eau potable.

\subsubsection{Chloration de sécurité}

Sans chlore, l'eau peut sortir stérilisée de la station de traitement mais, après son voyage souvent long dans le réseau de distribution, peut arriver contaminée au robinet du consommateur. La présence de chlore résiduel dans le réseau de distribution constitue une assurance qualité. De plus, le chlore peut permettre de détecter une contamination dont sa disparition brutale est le signe. En effet, toute substance organique introduite dans le réseau consomme immanquablement le chlore libre. 
D'ailleurs, en 1991 pendant la guerre du Golfe, l'objectif de teneur en chlore résiduel dans les réseaux de distribution, en particulier à Paris, avait été porté à $0,20-0,30 \mathrm{mg} / 1$ (contre $0,05-0,10 \mathrm{mg} / \mathrm{l}$ en temps normal). L'installation de microcapteurs de chlore libre dans le réseau de distribution permettra de constituer un réseau d'alerte en temps réel contre les contaminations, les mesures faites en continu par ces micro-capteurs étant transmises au centre de contrôle et de commande.

Il faut noter que les effets négatifs de la chloration (goût et formation d'organochlorés) peuvent être réduits, voire annulés, par les nouvelles techniques de traitement, qui réduisent les teneurs en matière organique dans les eaux potables : la préchloration est déjà très largement abandonnée, la filtration sur charbon actif se généralise et la nanofiltration se met au point.

Bien que certaines villes l'aient fait, comme Amsterdam, supprimer la chloration rend le réseau de distribution d'eau potable vulnérable, car il n'a plus alors la capacité de lutter contre les contaminations, accidentelles ou criminelles, et de les détecter. La potabilité de l'eau au robinet du consommateur n'est plus garantie en toutes circonstances.

\subsection{Les solutions de secours}

\subsubsection{Dispositifs de secours}

(i) - Un réseau d'alimentation en eau potable doit disposer de réservoirs tampons couvrant les besoins d'une journée en fonctionnement gravitaire.

(ii) - Les ressources en eau doivent être diversifiées et les réseaux interconnectés.

C'est ainsi que la région parisienne consomme 2 à 3 millions de mètres cubes par jour à partir des ressources suivantes :

- $60 \%$ proviennnent de trois rivières (Seine, Marne et Oise),

- $20 \%$ d'eaux souterraines,

- $20 \%$ d'eaux de source en adduction gravitaire $(60 \%$ pour Paris intra muros).

La capacité des interconnexions entre les divers réseaux de la région parisienne est de $1900000 \mathrm{~m}^{3 / j}$ jour. Ainsi une pollution accidentelle sur deux des trois rivières n'entraîne pas en général de perturbation dans la distribution, grâce à un appel renforcé aux autres ressources et éventuellement à l'activation des interconnexions.

(iii) - Des groupes électrogènes doivent pouvoir permettre de pallier aux coupures d'électricité. L'investissement en groupes électrogènes peut d'ailleurs être rentable, car ces groupes permettent d'obtenir d'E.D.F. le tarif E.J.P. (effacement des jours de pointe).

(iv) - La possibilité de fonctionner en manuel doit être préservée. C'est ainsi que le personnel de la SAGEP-Eau de Paris est équipé et entraîné pour cela (avec bicyclettes et talkies-walkies).

\subsubsection{Réseau de secours}

Un réseau de secours, distinct du réseau principal, peut permettre d'assurer l'alimentation de certains usagers prioritaires, tels que les hôpitaux, et un service minimum pour la population sur le mode aller-chercher.

Un tel réseau existe à Zurich : c'est un réseau alimenté gravitairement par des sources ; il dessert les hôpitaux et les abris, et des bornes-fontaines pouvant être équipées de robinets (1 robinet pour 100 habitants).

A Paris, la nappe profonde de l'Albien, à l'abri de toute contamination y compris radioactive, permettrait d'alimenter un tel réseau de secours, ou des unités d'ensachage, directement à partir des forages.
Les dispositifs ou les solutions de secours ne doivent pas être inventés pendant la crise ; ils doivent exister effectivement en réserve et pouvoir être activés rapidement.

\subsection{Les plans d'urgence}

C'est la responsabilité des distributeurs d'eau d'élaborer des plans de gestion de crise. Cependant, pour les cas où la crise dépasserait les capacités de gestion du distributeur, les pouvoirs publics doivent disposer de plans d'urgence. Ces plans d'urgence doivent :

- définir la responsabilité des acteurs (distributeur d'eau, municipalité, préfecture) et leurs contacts,

- recenser les usagers prioritaires,

- recenser les moyens de secours, faisant appel à la solidarité départementale, régionale, voire nationale,

- établir des fiches réflexes pour la mise en œuvre des secours,

- prévoir le cadre de la communication de crise.

Cependant, il faut être bien conscient qu'un plan d'urgence n'est opérationnel que s'il est tenu à jour et s'il fait l'objet d'exercices d'entraînement. Il n'est pleinement efficace que s'il est informatisé et s'appuie sur une banque de données et un système d'information géographique. Un plan modèle de gestion du risque eau potable a ainsi été élaboré dans le département du Tarn-et-Garonne et a permis la mise au point d'une méthodologie, susceptible d'être appliquée après ajustement dans les autres départements.

En cas de crise compromettant la potabilité de l'eau distribuée, l'expérience montre qu'au lieu de couper la distribution il vaut mieux, sauf risque toxique ou bactériologique grave, laisser le réseau sous pression, à condition d'avertir la population de ne pas boire l'eau du robinet et éventuellement de la colorer. Cela permet en particulier de continuer à assurer les besoins sanitaires et d'hygiène corporelle et le service incendie. La remise en service du service d'eau potable en est facilitée.

\subsection{Spécificités françaises en matière d'alimentation en eau potable}

Les unités de distribution d'eau potable ont en France une taille relativement limitée, avec un ratio moyen de seulement 4000 habitants par unité, contre par exemple 10000 en Allemagne.

Cette dispersion est compensée par l'existence de grandes sociétés privées d'importance nationale (et internationale) qui peuvent recevoir délégation des collectivités locales, maîtres d'ouvrage, pour assurer le service public de la distribution d'eau potable. Ces sociétés, qui sont la Générale des Eaux, la Lyonnaise des Eaux et la SAUR, desservent $75 \%$ de la population française, les $25 \%$ restants étant alimentés par des régies municipales. En cas de crise sur l'un des réseaux qu'ils gèrent, ces grands groupes peuvent mobiliser des moyens nationaux importants. C'est ainsi qu'en 1988 lors des inondations catastrophiques de Nîmes, qui ont mis hors d'usage le réseau d'eau potable de la ville (130 000 habitants desservis), la distribution a pu être rétablie, grâce à la mobilisation du groupe SAUR gestionnaire du réseau, à $75 \%$ après 2 jours et à $90 \%$ après 3 jours, avec une eau bactériologiquement potable.

Compte tenu des besoins en boisson couverts par les liquides embouteillés, la part de l'eau potable du robinet effectivement bue est faible :

- eaux minérales ou de table

- lait

— vin, bière et jus de fruit

$0,25 \mathrm{l} / \mathrm{j} /$ habitant

$0,20 \quad$ "

$0,40 \quad "$

Total boissons embouteillées

$0,85 \mathrm{l} / \mathrm{j} / \mathrm{habitant}$ 
Sur des besoins en boisson estimés à 2 litres par jour et par habitant, la part de l'eau potable du robinet est ainsi seulement de $1,15 \mathrm{l} / \mathrm{j} /$ habitant, ce qui, rapporté aux 250 litres d'eau potable consommés en France par jour et par habitant, représente seulement $0,5 \%$.

\subsection{Analyse d'une crise extrême en matière d'alimentation en eau potable : secours au camp de réfugiés du Rwanda en 1994}

\subsubsection{Situation}

En 1994, les camps de réfugiés du Rwanda regroupaient 1,2 million de réfugiés. Ils étaient situés près du Lac Kivu, il n'y avait donc pas de problème quantitatif d'eau, mais très vite le choléra s'est déclaré et a fait des milliers de morts.

\subsubsection{Opérations de secours}

Les distributeurs d'eau français (Générale des Eaux, Lyonnaise des Eaux, SAUR, SADE et CISE), se sont mobilisés avec l'aide de l'armée française présente sur place. Le 24 juillet 1994, 14 techniciens et 30 tonnes de matériels sont acheminés par air. L'opération consiste :

- d'abord, à assurer l'alimentation en eau directement à partir du lac par l'installation de groupes de pompage et de bâches de stockage avec dispositif de chloration (capacité installée : $7600 \mathrm{~m}^{3} /$ jour),

- ensuite, à remettre en marche (en les doublant) les deux réseaux d'eau potable existants des villes de Cyangugu et de Goma (capacité installée : $4500 \mathrm{~m}^{3} /$ jour).

\subsubsection{Résultat:}

Après 3 semaines, 12 millions de litres par jour d'une eau désinfectée et donc saine pouvaient ainsi être distribués, permettant de subvenir aux besoins de 1,2 million de réfugiés, à raison de 10 litres par jour et par personne.

\subsubsection{Coût}

5 millions de francs qui ont permis de sauver des dizaines de milliers de vies.

\section{- 4.8 Conclusion eau potable}

La sécurité d'un réseau d'eau potable ne s'improvise pas. L'équipement des réseaux doit intégrer cet objectif de sécurisation, qui se concrétise par une obligation de résultat, c'està-dire de niveau de service minimal en cas de crise. Rappelons que le service minimum qu'il est souhaitable de maintenir ou de rétablir rapidement pour assurer un niveau d'activité économique acceptable est de 100 litres par jour et par habitant. Il appartient aux collectivités et aux gestionnaires de réseaux de choisir les moyens optimaux pour assurer en toute circonstance le niveau de service nécessaire. Nous sommes entrés dans une civilisation à risques et l'eau est au cœur de tous les dangers.

\section{V ロ CRISE EXTRÊME À CONSÉQUENCES MULTIPLES}

La crue de type 1910 à PARIS entre dans la catégorie des crises extrêmes à conséquences multiples. Les dernières crues centennales de la Seine datent de 1740 et de 1910. A quand la prochaine ?

1) La Seine à Paris a un bassin versant de $43000 \mathrm{~km}^{2}$ avec un débit moyen de $260 \mathrm{~m}^{3} / \mathrm{s}$ (dont 1/3 provient de la Marne).
Au delà de $1000 \mathrm{~m}^{3} / \mathrm{s}$ (crue mineure), il y a inondation des voies sur berge.

2) Les dernières crues majeures par ordre d'importance croissante (hauteur en mètres atteinte au pont d'Austerlitz) sont :

- $1982(6,18 \mathrm{~m})$ avec fermeture de la ligne C du RER

- $1955(7,14 \mathrm{~m})$ crue cinquentennale (dégâts estimés à 16,5 Milliards F)

- $1910(8,62 \mathrm{~m})$ crue centennale prise comme crue de référence $\left(2500 \mathrm{~m}^{3} / \mathrm{s}\right.$ et un volume écoulé de 3 à 4 milliards $\left.\mathrm{m}^{3}\right)$. 3) Paris et la région parisienne sont à la merci d'une crue centennale de type 1910 dont les conséquences catastrophiques seraient les suivantes :

- Dégâts (directs + indirects) estimés à 60 Milliards de F,

- 25000 hectares inondés, dont 1250 à Paris,

- Désorganisation complète de la vie dans la capitale (électricité, téléphone, eau potable, chauffage urbain, ravitaillement, collecte des ordures ménagères, transports...). Ainsi la RATP ne fonctionnerait qu'à $30 \%$ du trafic normal pendant une durée d'au moins 2 mois. Le trafic banlieue serait interrompu sur les gares de Lyon et d'Austerlitz.

4) Il faut être bien conscient que les grands lacs de Seine, dont la capacité de stockage est de 830 millions de $\mathrm{m}^{3}$, ne mettent pas la région parisienne à l'abri d'une crue de type 1910. Ces grands lacs ne contrôlent en effet que $17 \%$ des apports. Les crues du Loing et des Morins en particulier ne sont pas affectées par les grands lacs de Seine. La crue de 1910 n'aurait n'aurait été que peu écrêtée par ces barragesréservoirs $(0,80 \mathrm{~m}$ de moins au pont d'Austerlitz).

Sur l'Yonne, la zone humide de la Bassée est actuellement à l'étude pour le stockage de 50 millions de $\mathrm{m}^{3}$ supplémentaires.

Si les grands lacs de Seine et le creusement du lit de la Seine pour la navigation sont effectivement des aménagements positifs, l'augmentation de l'urbanisation et donc des coefficients d'écoulement dans le bassin versant constitue un facteur d'aggravation de la crue.

5) L'eau potable à Paris en cas de crue de type 1910.

Compte tenu des ouvrages inondés et de la turbidité de la Marne et des sources de l'Avre, seuls resteraient en production l'usine d'Orly et les aqueducs de la Vanne et du Loing, soit une production totale d'eau potable de $550000 \mathrm{~m}^{3} / \mathrm{j}$ ou $80 \%$ de la demande journalière moyenne. Ce niveau de service est satisfaisant dans le contexte d'une telle crise et ne devrait pas poser de problèmes importants. Des problèmes localisés pourraient cependant apparaitre au niveau du réseau de distribution : les galeries d'égout, dans lesquelles sont fixées les conduites d'eau potable à Paris, seront noyées, donc ne seront plus visitables.

6) Cette crue de type 1910 peut arriver "demain". Elle coûtera cher, sera catastrophique pour la vie parisienne et affectera la nation toute entière.

Il faut donc poursuivre les mesures de protection.

\section{VI $\square$ INFORMATION DES POPULATIONS ET DÉCLENCHEMENT DE L'ALERTE}

Les conséquences d'une crise de type "sécurité civile" peuvent être fortement réduites si la population est informée de l'existence du risque et si elle sait quoi faire, c'est-à-dire si elle a les bons réflexes lorsque la crise survient. C'est l'information préventive des populations avec affichage des actions réflexes.

Dans chaque département a été créée une cellule d'analyse des risques et d'information préventive (CARIP). Cette cellule élabore un dossier départemental des risques majeurs comprenant la liste des communes exposées à chaque risque particulier. Sont élaborés ensuite les dossiers communaux. Les affiches correspondant aux divers risques aux- 
quels chaque commune est exposée sont mises au point et affichées en mairie et sur les sites exposés.

Le déclenchement de l'alerte doit se faire par un réseau d'alerte opérationnel et efficace, qui en France demande à être rénové, en particulier en ce qui concerne le réseau de sirènes. Le système classique d'annonce de crues est insuffisant pour les crues "flash" de type méditerranéen qui nécessitent pour la protection de la population une couverture par radars pluviométriques, en cours d'installation progressive sur les zones à risques. Compte tenu des faibles temps de concentration sur les petits bassins avec pentes fortes, l'alerte doit être donnée au stade des précipitations avant que cellesci ne donnent naissance à l'écoulement de crue. Encore fautil que la détection des précipitations intenses par le radar pluviométrique soit exploitée en temps réel par quelqu'un qui décide de lancer l'alerte.

\section{VII $\square$ PLAN DE PRÉVENTION DES RISQUES PRÉVISIBLES (P.P.R.)}

Les Plans de Prévention des Risques prévisibles (P.P.R.) ont été créés par la loi du 2 février 1995 relative aux renforcements de la protection de l'environnement. Ils remplacent les Plans d'Exposition aux Risques (P.E.R.).

Les P.P.R. délimitent les zones exposées et définissent les mesures de prévention, de protection et de sauvegarde et les contraintes d'aménagement.

Les P.P.R. sont élaborés par les services de l'Etat et approuvés par les préfets après enquête publique. Ils valent servitude d'utilité publique et sont annexés aux Plans d'Occupation des Sols (P.O.S.). 2000 communes, particulièrement exposées aux risques naturels, doivent être dotées en priorité d'un P.P.R.

Dans le cas de risques naturels prévisibles menaçant gravement des vies humaines, les biens exposés peuvent faire l'objet d'une expropriation.

\section{VIII - MENACES GLOBALES AU NIVEAU PLANÉTAIRE}

La dégradation de l'environnement planétaire pourrait provoquer une crise écologique mondiale. Nous en observons certains prémisses : 1995 a été une année noire avec cyclones, inondations et tremblements de terre catastrophiques.
Les vingt dernières années ont vu dans le monde 3 millions de morts et 1 milliard de sans-abri par catastrophes naturelles. Le nombre de ces morts semble augmenter de $50 \%$ tous les 10 ans depuis 1950. Les personnes affectées par les catastrophes naturelles augmenteraient de $6 \%$ par an, soit 3 fois la croissance démographique.

Le coût de ces catastrophes naturelles a été multiplié par 3 en 30 ans. Il atteint 220 milliards de francs en 1989, s'est élevé à 300 milliards de francs en 1992 et pourrait atteindre 500 milliards de francs en l'an 2000. Ces catastrophes naturelles sont en partie le fait de l'homme à cause de la concentration urbaine, de la désertification rurale et peut-être des débuts de l'effet de serre.

Outre ces morts et ces dégâts, les catastrophes naturelles ont engendré un genre nouveau de réfugiés : les réfugiés de l'environnement qui, chassés par les sécheresses, les inondations, sont plus de 25 millions, dépassant ainsi la vingtaine de millions de réfugiés classiques (politiques, sociaux, ethniques ou religieux).

Les incendies de forêts de septembre et octobre 1997 en Indonésie ont entraîné une pollution de l'air qui a affecté une partie importante du Sud-Est asiatique.

\section{IX $\square$ CONCLUSION}

Une science nouvelle est née : les Cindyniques ou sciences du danger (du grec Kindunos : Danger). Cette science a pour but de nous aider à faire face aux crises, à affronter des ruptures, à analyser et gérer les comportements et les peurs individuelles face aux dangers. Les Cindyniques concrétisent notre rejet du concept de fatalité : il s'agit de passer de l'âge du sang et de l'âge des larmes à l'âge des neurones.

\section{BIBLIOGRAPHIE}

[1] M. Defourneaux. "Guerre des armes, guerre des hommes", Editions ADDM, (Paris 1994)

[2] P. LAGADEC. "Apprendre à gérer les crises", Editions ORGANISATION, (Paris 1993)

[3] G. Y. KeRVEn et P. RUBISE, "L'archipel du danger - Introduction aux cindyniques", Editions ECONOMICA (Paris). 